Open Access

\title{
Spontaneous regression of non-small cell lung cancer after biopsy of a mediastinal lymph node metastasis: a case report
}

\author{
Alberto Lopez-Pastorini ${ }^{1 *}$, Till Plönes ${ }^{1}$, Michael Brockmann ${ }^{2}$, Corinna Ludwig ${ }^{1}$, Frank Beckers ${ }^{1}$ and Erich Stoelben ${ }^{1}$
}

\begin{abstract}
Introduction: Spontaneous regression of cancer is defined as a complete or partial, temporary or permanent disappearance of tumor in the absence of specific therapy. With only a few cases reported, spontaneous regression is extremely rare in primary lung cancer. Regarding spontaneous regression in lung cancer, recent investigations revealed the role of immunological mechanisms, thus indicating potential treatment options by specific immunotherapy in the future.
\end{abstract}

Case presentation: A 76-year-old Caucasian man with progressive dyspnea presented to our hospital. A computed tomography scan revealed a tumor mass in the upper lobe of his right lung and enlarged mediastinal lymph nodes. A biopsy of a paratracheal lymph node by mediastinoscopy disclosed metastatic lung cancer. By immunohistochemical findings the tumor was classified as large cell carcinoma. Diagnosed with clinical stage IIIA non-small cell lung cancer, a neoadjuvant therapy concept was indicated. However, before starting chemoradiation, a computed tomography scan showed a regression of both the tumor mass in the upper lobe of his right lung and the mediastinal lymphadenopathy. As a repeated computed tomography scan showed further regression, we agreed with our patient to perform routine follow-up instead of starting therapy. To date, no relapse has been reported.

Conclusions: Given the circumstances that regression started after the biopsy and involved both the tumor in the upper lobe of his right lung and the mediastinal lymph node metastases, an immune response is a reasonable explanation for the observed spontaneous regression in this case.

Keywords: Spontaneous regression, Immunologic reaction, Non-small cell lung cancer, Biopsy, Complete remission

\section{Introduction}

Spontaneous regression (SR) of cancer is an unusual event and extremely rare in primary lung cancer. It is defined as a complete or partial, temporary or permanent disappearance of tumor in the absence of anticancer therapy. Although the concrete mechanisms of SR remain unknown, recent investigations revealed the role of immunological mechanisms involved in SR of lung cancer.

Here we report the case of a patient with an advancedstage non-small cell lung cancer (NSCLC) that completely

\footnotetext{
*Correspondence: Alberto.Lopez@uni-wh.de

'Department of Thoracic Surgery, University Medical Center Witten/Herdecke, Lung Clinic Merheim, Campus Cologne, Ostmerheimerstr. 200, 51109

Cologne, Germany

Full list of author information is available at the end of the article
}

regressed after a biopsy of a mediastinal lymph node metastasis.

\section{Case presentation}

A 76-year-old Caucasian man with progressive dyspnea for the last two months was admitted to our hospital. A contrast-enhanced computed tomography (CT) scan of his chest disclosed an oval-shaped tumor mass in the upper lobe of his right lung, adjoining the pleura and $6 \times$ $5 \times 3 \mathrm{~cm}$ in size (Fig. 1a). In addition, the CT scan showed enlarged mediastinal lymph nodes in the right paratracheal position. Our patient was an active smoker with a cumulative exposure of 50 pack-years and had a medical history of hypertension and hyperlipidemia. His actual medication consisted of an ACE inhibitor and a statin with no changes for the last 2 years. Physical and laboratory examinations revealed no abnormal findings. A 

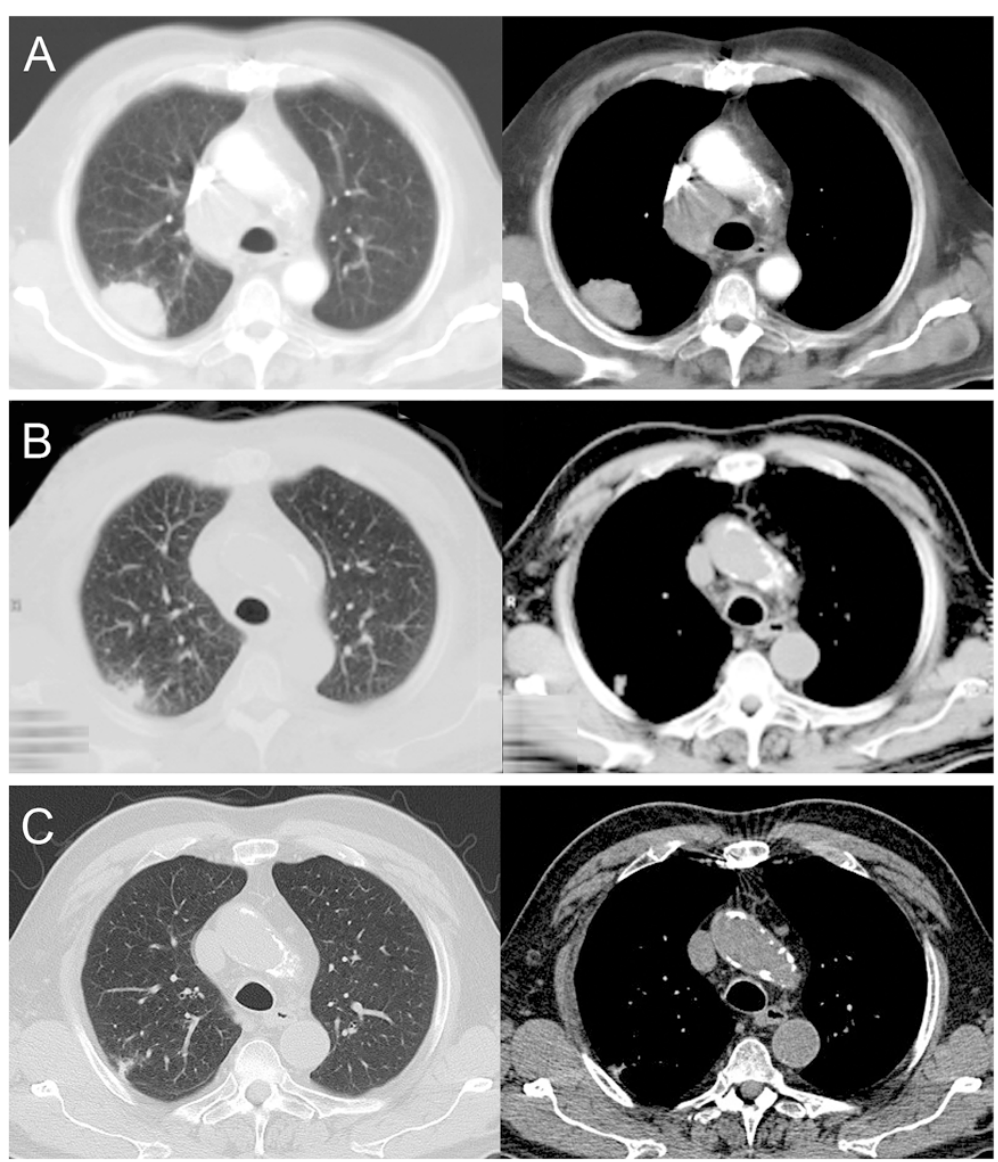

Fig. 1 a Initial chest computed tomography scan showing the tumor in the upper lobe of the right lung and enlarged mediastinal lymph nodes in the paratracheal position. b Computed tomography scan after 2 months, c after 1 year

mediastinoscopic biopsy of the mediastinal lymph nodes revealed metastatic cells of a poorly differentiated NSCLC in a paratracheal lymph node (4R) (Fig. 2). Immunohistochemical findings showed positive staining for cytokeratin (CK) 7 but no reactivity with antibodies against TTF1, CK5/6, p63 and napsin. Furthermore the cells were negative for CD56, chromogranin and synaptophysin. By these findings, the tumor was classified as large cell carcinoma. Further staging procedures including bone scintigraphy, abdominal CT and head magnetic resonance imaging (MRI) disclosed no distant metastases, so that our patient was diagnosed with clinical stage IIIA (T2bN2M0) NSCLC. Consequently, a neoadjuvant concept consisting of a combined chemoradiation was indicated.

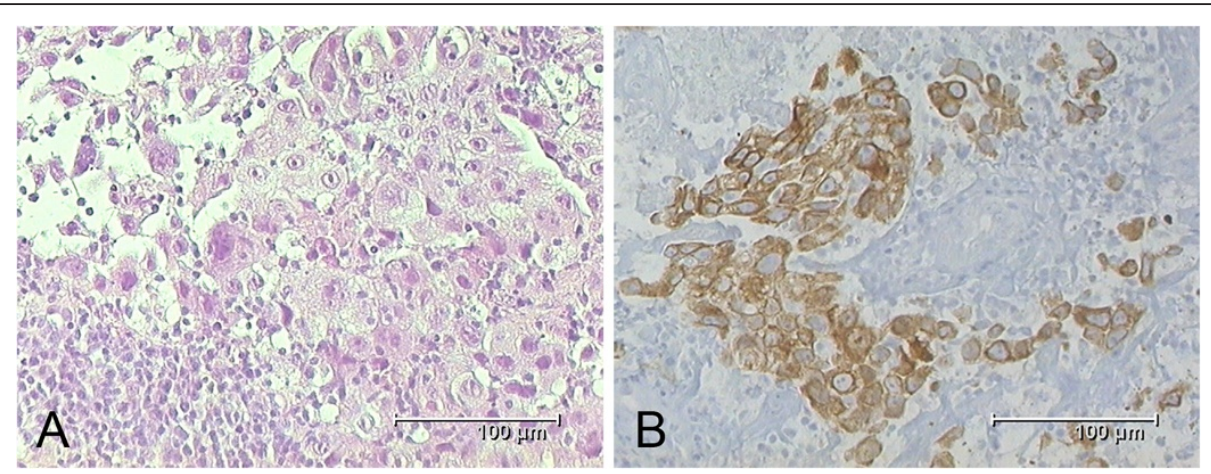

Fig. 2 a Tissue obtained by biopsy of a right paratracheal lymph node showing metastatic cells of large cell carcinoma. b Immunohistochemical staining showing cytokeratin positivity (KL-1) of tumorous cells within the lymphatic tissue 
However, CT planning before starting therapy and 2 weeks after mediastinoscopy showed a decrease of both the tumor mass in the upper lobe of his right lung and the mediastinal lymph nodes. We conducted a CTguided fine-needle biopsy of the tumor in the upper lobe of his right lung (Fig. 3). The histological examination showed extended necrosis but failed to prove malignant cells. In addition, the tissue obtained by mediastinoscopy was examined by a second pathologist, who confirmed the prior diagnosis of NSCLC. A repeated chest CT scan showed further regression of the tumor and the mediastinal lymphadenopathy (Fig. 1b). At this time we agreed with our patient to postpone chemoradiation and instead perform a routine follow-up by an annual chest CT scan. After 1 year, a CT scan revealed the almost complete disappearance of the tumor in the upper lobe of his right lung and a decrease of the mediastinal lymph nodes to normal size (Fig. 1c). Up to the present day, our patient has received no anticancer therapy. He remains in follow-up care in our hospital and after 7 years no relapse has been reported.

\section{Discussion}

The most commonly accepted criteria for spontaneous regression (SR) of cancer were postulated by Everson and Cole in 1959. Here SR is defined as the partial or complete, temporary or permanent, disappearance of the tumor in the absence of any specific therapy [1]. The actual incidence of spontaneous regression is unknown [2]. By the Everson and Cole definition, it was estimated to be not more than 1 in 60,000 to 100,000 cases [3].

Compared with other solid tumors in which SR is more frequently reported, like renal cell carcinoma or malignant melanoma, SR of primary lung cancer is

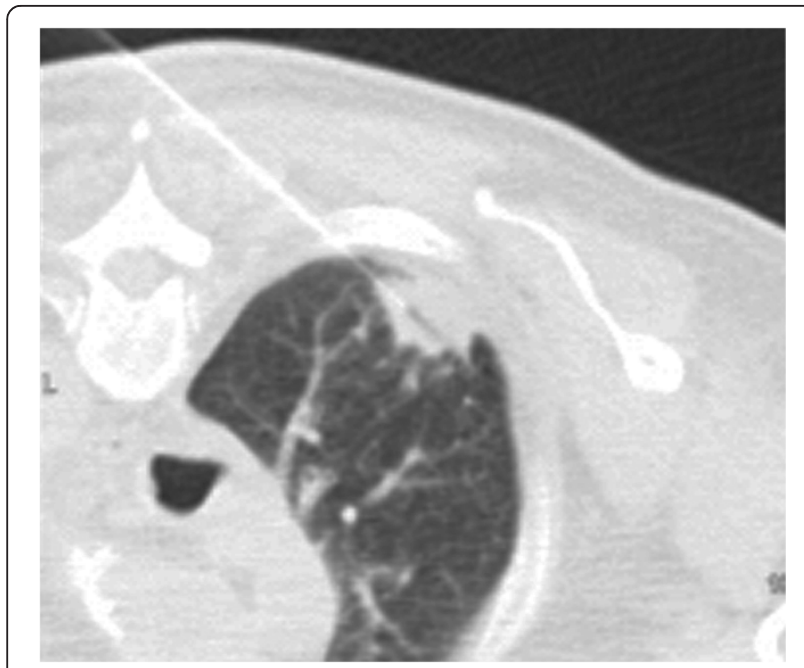

Fig. 3 Computed tomography-guided fine-needle biopsy of the tumor in the upper lobe of the right lung extremely rare [2, 4]. Kumar and colleagues list only two cases of primary lung cancer from 1951 to 2008 that meet the definition of Everson and Cole [5].

There are several suggested mechanisms including immunological factors, hormonal changes, trauma or variations in blood supply $[2,4,6]$.

Once regarded as a poorly immunogenic tumor, NSCLC has recently emerged as a target for promising cancer vaccines and immune modulators [7, 8]. Nivolumab, a monoclonal antibody for the human programmed cell death 1 (PD-1) receptor, has been approved this year in the United States for the treatment of squamous NSCLC. In a phase I clinical trial of nivolumab in $201233 \%$ of the squamous cell lung cancers and $12 \%$ of the nonsquamous cell tumors responded with an overall median duration of response of 74 weeks [7]. Interestingly, a smoking history has been associated with an improved response to PD-1 blockade due to a higher neoantigen burden in smokers [9].

Referring to lung cancer, recent articles describe changes in the immunological environment of the tumor that can affect both oncogenesis and regression. Scheider et al. showed an accumulation of regulatory $\mathrm{T}$ cells in pulmonary adenocarcinoma and metastatic lymph nodes, resulting in a local decrease of antitumor immune response by natural killer cells [10]. Iwakami and colleagues reported an infiltration of CD8-positive lymphocytes in small cell lung cancer that regressed spontaneously, indicating that $\mathrm{T}$ cell-mediated cytotoxity is a possible mechanism of SR in lung cancer [11]. Isobe et al. demonstrated an integrated immune response consisting of immunoglobulin G (IgG) antibodies, CD4 and CD8 T cells against a NY-ESO-1-expressing NSCLC experiencing SR [12].

Several cases of SR have implicated surgery or biopsy conducted on the primary tumor or the metastases as elements that can induce an immunological response [13]. Cole reported that 71 out of 176 cases of SR in cancer were associated with some type of operative trauma $[3,14]$. This may be relevant to our patient, who received a biopsy of metastatic lymph nodes before SR was noted. The biopsies were performed with endoscopic forceps, this causing a disruption of the lymph nodes, potentially leading to a release of antigens with activation of the immune system.

There are two important limitations in our case that need to be discussed. In the first place, we were not able to prove malignancy of the tumor in the upper lobe of the right lung. Histological examinations showed extensive necrosis, potentially caused by tumor disintegration. However, being a peripheral lesion, also a lung infarction, an organizing pneumonia or a limited pulmonary vasculitis should also be considered as possible cause. Immunohistochemical analyses to distinguish tumor 
necrosis from such an infarction, for example by reticulin or keratin staining, were not performed. In conclusion, there is no certain evidence of a primary tumor in this case. Second, by microscopic and immunohistochemical findings, the tumor cells in the paratracheal lymph node were classified as large cell carcinoma. This is rather a diagnosis of exclusion and does not prove a malignancy of the lung exclusively. Carcinomas of several origins and entities show CK7 positivity, so that in theory, this could be a metastasis from elsewhere in combination with a lung infarction.

Summing up our findings, we had a highly suspicious pulmonary lesion with a proven metastatic lymph node in an expectable position for lung cancer. In addition, neither did the staging procedures reveal any other lesions nor did a malignancy develop elsewhere in 7 years of follow-up. Therefore, in our opinion, the most reasonable explanation for the findings in our case is a primary lung cancer with lymph node metastases.

\section{Conclusions}

The present case complies with the above mentioned criteria of SR. In our case, cells of a large cell carcinoma were proven by pathological findings in a mediastinal lymph node metastasis. Although our patient received no treatment, a near complete remission could be observed within 1 year. Given the circumstances that the regression started after the biopsy and involved both the tumor in the upper lobe of his right lung and the mediastinal lymph node metastases, an immune response is a reasonable explanation for the observed SR in this case.

\section{Consent}

Written informed consent was obtained from the patient for publication of this case report and any accompanying images. A copy of the written consent is available for review by the Editor-in-Chief of this journal.

\section{Abbreviations}

CT: computed tomography; CK: cytokeratin; lgG: immunoglobulin G; MRI: magnetic resonance imaging; NSCLC: non-small cell lung cancer; PD-1: human programmed cell death 1; SR: spontaneous regression.

\section{Competing interests}

This study was not financed by any science foundation or support program. The authors have no competing interests, including relevant financial interests, activities, and affiliations.

\section{Authors' contributions}

ALP conceptualized and wrote the case report. TP edited the manuscript as a major contributer. ES and CL edited the manuscript and were involved in caring for the patient. MB carried out the histopathologic examination and immunohistochemical study. FB was involved in treatment during hospitalization and revising the manuscript for intellectual content. All authors read and approved the final manuscript.

\section{Acknowledgements}

We wish to thank Kate and Michael Szczepanski for their critical review of the manuscript. Neither author received any source of funding for this manuscript.

\section{Author details}

${ }^{1}$ Department of Thoracic Surgery, University Medical Center Witten/Herdecke, Lung Clinic Merheim, Campus Cologne, Ostmerheimerstr. 200, 51109

Cologne, Germany. ²Department of Pathology, University Medical Center Witten/Herdecke, Clinic Merheim, Campus Cologne, Ostmerheimerstr. 200, 51109 Cologne, Germany.

Received: 10 February 2015 Accepted: 31 August 2015

Published online: 17 September 2015

\section{References}

1. Everson TC, Cole WH. Spontaneous regression of malignant disease. J Am Med Assoc.1959; 169:1758-9.

2. Papac RJ. Spontaneous regression of cancer. Cancer Treat Rev. 1996; 22:395-423.

3. Cole WH. Efforts to explain spontaneous regression of cancer.J Surg Oncol. 1981: 17:201-9.

4. Cole WH. Relationship of causative factors in spontaneous regression of cancer to immunologic factors possibly effective in cancer. J Surg Oncol. 1976; 8:391-411

5. Kumar T, Patel N, Talwar A. Spontaneous regression of thoracic malignancies. Respir Med. 2010; 104:1543-50.

6. Chang WY. Complete spontaneous regression of cancer: four case reports, review of literature, and discussion of possible mechanisms involved. Hawaii Med J. 2000; 59:379-87.

7. Sundar R, Cho BC, Brahmer JR, Soo RA. Nivolumab in NSCLC: latest evidence and clinical potential. Ther Adv Med Oncol.2015; 7(2):85-96.

8. Brahmer JR, Pardoll DM. Immune checkpoint inhibitors: making immunotherapy a reality for the treatment of lung cancer. Cancerlmmunol Res. 2013; 1(2):85-91.

9. Rizvi NA, Hellmann MD, Snyder A, Kvistborg P, Makarov V, Havel JJ et al. Cancer immunology. Mutational landscape determines sensitivity to PD-1 blockade in non-small cell lung cancer. Science. 2015; 348(6230):124-8.

10. Schneider T, Kimpfler S, Warth A, Schnabel PA, Dienemann H, Schadendorf D, et al. Foxp3(+) regulatory T cells and natural killer cells distinctly infiltrate primary tumors and draining lymph nodes in pulmonary adenocarcinoma. J Thorac Oncol. 2011; 6:432-8.

11. Iwakami S, Fujii M, Ishiwata T, Iwakami N, Hara M, Ihara H, et al. Small-cell lung cancer exhibiting spontaneous regression. Intern Med. 2013; 52:2249-52.

12. Isobe M, Eikawa S, Uenaka A, Nakamura Y, Kanda T, Kohno S, et al. Correlation of high and decreased NY-ESO-1 immunity to spontaneous regression and subsequent recurrence in a lung cancer patient. Cancer Immun. 2009; 9:8.

13. Challis $\mathrm{GB}$, Stam $\mathrm{HJ}$. The spontaneous regression of cancer. A review of cases from 1900 to 1987. Acta Oncol. 1990; 29:545-50.

14. Cole WH. Spontaneous regression of cancer: the metabolic triumph of the host? Ann N Y Acad Sci. 1974; 230:111-41.

\section{Submit your next manuscript to BioMed Central and take full advantage of:}

- Convenient online submission

- Thorough peer review

- No space constraints or color figure charges

- Immediate publication on acceptance

- Inclusion in PubMed, CAS, Scopus and Google Scholar

- Research which is freely available for redistribution 